\title{
Dynamics of quasi-perpendicular shocks: Recent results issued from 2D PIC simulation
}

\author{
$\underline{\text { Takayuki Umeda }^{1}, \text { Yoshitaka Kidani }^{I} \text {, and Shuichi Matsukiyo }}{ }^{2}$ \\ ${ }^{1}$ Solar-Terrestrial Environment Laboratory, Nagoya University, Nagoya, Aichi 464-8601, Japan \\ umeda@stelab.nagoya-u.ac.jp; kidani@stelab.nagoya-u.ac.jp \\ ${ }^{2}$ Earth System Science and Technology, Kyushu University, Kasuga, Fukuoka 816-8580, Japan \\ matsukiy@esst.kyushu-u.ac.jp
}

\begin{abstract}
Cross-scale coupling between fluid dynamics and particle kinetics at perpendicular collisionless shocks is an issue of space plasma physics. The influence of shock-front ripples to the dynamics of shocks is studied by means of a large-scale two-dimensional (2D) full particle-in-cell (PIC) simulation. The present simulation has confirmed the transition of shock structures from the cyclic self-reformation to the quasi-stationary shock front due to rippled structures at the shock front.
\end{abstract}

\section{Introduction}

Recent development of supercomputer technology allows us to perform large-scale plasma simulations. Largescale kinetic simulations have confirmed the influence of particle kinetics on the structure and dynamics of collisionless shocks. Reflection of ions at the shock front is responsible for the periodic appearance and disappearance of a new shock front on a timescale of the ion cyclotron period, which is known as the shock reformation. There also appear fluctuations with a large amplitude at the shock front on the spatial scale of ion inertial length in the shock-tangential direction, which is known as ripples. Recent two-dimensional (2D) hybrid particle-in-cell (PIC) simulations obtained a contradictory result. Hellinger et al. [1] reported that the periodic reformation of shock front is suppressed by excitation of whistler mode waves at an exactly-perpendicular shock, while Yuan et al. [2] found the shock reformation at a quasiperpendicular shock. The suppression of the shock reformation has also confirmed in recent large-scale 2D full PIC simulations $[3,4]$. Although the previous hybrid simulations used the very similar simulation parameters, the obtained results seem to differ substantially. In the present study, we performed a 2-D full PIC simulation of both quasi- and exactly-perpendicular shocks in order to make direct comparison between them.

\section{Method}

We developed a new method for exciting collisionless shocks in kinetic plasma simulations, where the simulation system is taken in the shock rest frame [5-7]. The new method allows us to study longer-time development of collisionless shocks on a limited computer resource. Our simulation code is a standard full PIC code where the Maxwell equations, the equations of motion for individual charged particles, and the charge continuity equations are solved in self-consistent manner.

\section{Result and Summary}

We confirmed that both (quasi- and exactly-perpendicular shock) simulations give similar results. The shock magnetic field averaged over the shock-tangential direction shows that the shock reformation takes place in an early phase but is suppressed in a later phase in which the shock-front ripples are strongly enhanced, which is consistent with Refs. $[1,3,4]$. On the other hand, local shock magnetic fields show evident shock reformation. The period of the shock reformation in the early phase corresponds to several ion cyclotron periods, but the reformation period is modified by the excitation of strong ripples in the later phase, which is consistent with Refs. [2,4].

We reached a simple conclusion that both previous works obtained similar results but analyzed them from different points of view. That is, Hellinger et al. [1] and Lembege et al. [3] analyzed the averaged shock magnetic field and found the transition from the reformation phase to the no-reformation phase, while Yuan et al. [2] analyzed local 
shock magnetic fields and found the modification of the reformation period during the transition. The present simulation results of both quasi-perpendicular and exactly perpendicular shocks are in agreement with these previous results [8].

\section{Acknowledgments}

This work was supported by Grant-in-Aid for Scientific Research on Innovative Areas 21200050. The computer simulations were performed on the Fujitsu FX1 and HX600 systems at the Information Technology Center, Nagoya University. This work was carried out as a computational joint research program at the Solar-Terrestrial Environment Laboratory, Nagoya University.

\section{References}

1. P. Hellinger, P. M. Travnicek, B. Lembege, and P. Savoini, Emission of nonlinear whistler waves at the front of perpendicular supercritical shocks: hybrid versus particle simulations, Geophysical Research Letters, 34, 2007 , L14109.

2. X. Yuan, I. H. Cairns, L. Trichtchenko, R. Rankin, and D. W. Danskin, "Confirmation of quasi-perpendicular shock reformation in twodimensional hybrid simulations," Geophysical Research Letters, 36, 2009, L05103.

3. B. Lembege, P. Savoini, P. Hellinger, and P. M. Travnicek, "Nonstationarity of a two-dimensional perpendicular shock: Competing mechanism," Journal of Geophysical Research, 114, 2009, A03217.

4. T. Umeda, M. Yamao, and R. Yamazaki, "Cross-scale coupling at a perpendicular collisionless shock," Planetary and Space Science, in press.

5. T. Umeda and R. Yamazaki, "Particle simulation of a perpendicular collisionless shock: A shock-rest-frame model," Earth, Planets and Space, 58, 2006, pp.e41-e44.

6. T. Umeda, M. Yamao, and R. Yamazaki, "Two-dimensional full particle simulation of a perpendicular collisionless shock with a shock-rest-frame model," Astrophysical Journal Letters, 681, 2008, pp.L85-L88.

7. T. Umeda, M. Yamao, and R. Yamazaki, "Electron acceleration at a low-Mach-number perpendicular collisionless shock," Astrophysical Journal, 695, 2009, pp.574-579.

8. T. Umeda, Y. Kidani, M. Yamao, S. Matsukiyo, and R. Yamazaki, "On the reformation at quasi- and exactly perpendicular shocks: Full particle-in-cell simulations," Journal of Geophysical Research, 115, 2010 , A10250. 